\title{
Diversity of the epidemiology of carbapenemase- producing Enterobacteriaceae in long-term acute care rehabilitation settings from an area of hyperendemicity, and evaluation of an intervention bundle
}

\author{
F. Arena ${ }^{\text {a }}$, F. Vannetti ${ }^{\mathrm{b}}$, V. Di Pilato ${ }^{c}$, L. Fabbri ${ }^{\mathrm{b}}$, O.L. Colavecchio ${ }^{\mathrm{c}}$, T. Giani $^{\mathrm{c}}$,

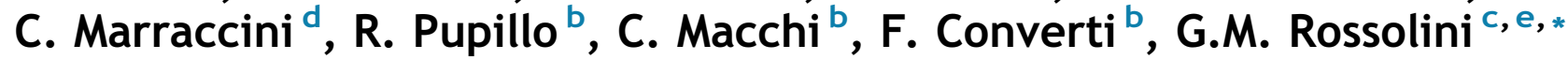 \\ a Department of Medical Biotechnologies, University of Siena, Siena, Italy \\ b IRCCS Fondazione Don Carlo Gnocchi, Florence, Italy \\ 'Department of Experimental and Clinical Medicine, University of Florence, Florence, Italy \\ d Synlab Laboratory, Sesto Fiorentino, Italy \\ e Clinical Microbiology and Virology Unit, Florence Careggi University Hospital, Florence, Italy
}

\section{A R T I C L E I N F O}

\section{Article history:}

Received 20 March 2018

Accepted 30 May 2018

Available online 4 June 2018

\section{Keywords:}

Klebsiella pneumoniae

KPC

Intestinal colonization

Infection control

\begin{abstract}
$S U M M A R Y$
Background: Long-term acute care rehabilitation facilities (LTACRFs) are affected by carbapenem-resistant Enterobacteriaceae (CRE) in endemic areas. However, the contribution of different subpopulations of patients has not been investigated in these settings. Aim: To study the epidemiology of CRE in an LTACRF, and the effect of an infection control intervention.

Methods: A surveillance programme was implemented in a large Italian LTACRF. The intervention included screening for CRE carriage at admission and weekly (for negative patients), and enforcement of contact precautions plus cohorting (in wards and rehabilitation areas) for presumed and confirmed carriers. Prevalence and incidence of CRE colonization and the number of CRE bacteraemias were monitored over one year.

Findings: Overall, 1084 patients underwent screening (adherence $89.8 \%$ ). At admission, $11.6 \%$ of patients were colonized, and $9.9 \%$ of those negative at admission subsequently became colonized. These percentages were significantly higher among patients with severe brain injuries (SBIs) who were exposed to a higher intensity of care $(44.1 \%$ vs $8.6 \%$ and $63.5 \%$ vs $6.8 \%$, respectively). The majority of CRE bacteraemias occurred in the SBI ward. The intervention was associated with a decline in the incidence of CRE colonization in the SBI ward (from 17.7 to 7.2 acquisitions/100 at-risk patient-weeks), but not in other wards. All CRE isolates were Klebsiella pneumoniae carbapenemase-producing K. pneumoniae.
\end{abstract}

\footnotetext{
* Corresponding author. Address: Dipartimento di Medicina Sperimentale e Clinica, Università di Firenze, S.O.D. Microbiologia e Virologia, Azienda Ospedaliera-Universitaria Careggi, Largo Brambilla, 50134 Florence, Italy.

E-mail address: gianmaria.rossolini@unifi.it (G.M. Rossolini).
} 
Conclusions: A peculiar CRE epidemiology was observed in a LTACRF from Italy, with very high rates of carriage and cross-transmission in SBI patients. A simplified infection control bundle was effective at reducing the incidence of CRE colonization in the SBI ward.

(C) 2018 The Healthcare Infection Society. Published by Elsevier Ltd. All rights reserved.

\section{Introduction}

The dissemination of carbapenem-resistant Enterobacteriaceae (CRE) has become a global problem and of the highest priority for public health [1]. CRE can spread rapidly in healthcare settings, and cause infections associated with high mortality rates due to limited antibiotic treatment options [2-4]. CRE infections are often preceded by carriage, and carriers represent the most important source for dissemination in the hospital setting $[5,6]$. Carriage can last for several months [7-9], and screening for intestinal carriage and contact isolation of colonized/infected patients have become a standard of care for infection control of CRE $[10,11]$.

In endemic areas, dissemination of CRE has mainly involved hospital settings, among which long-term acute care rehabilitation facilities (LTACRFs, i.e. acute and postacute care rehabilitation facilities with a mean length of stay $\geq 25$ days) bear a higher risk for CRE dissemination [12-17]. In fact, due to the peculiar organizational and logistical features (e.g. presence of common gyms and spaces), the implementation of infection control practices in LTACRFs can be particularly challenging. Recent studies have expanded the knowledge on the burden of CRE in LTACRFs, and described containment strategies [14-17]. However, knowledge of CRE epidemiology in these settings remains limited, and previous studies have not evaluated the involvement of different subpopulations of patients admitted to LTACRFs.

In Italy, an epidemic spread of CRE has been observed since 2010, with Klebsiella pneumoniae producing KPC-type carbapenemases (KPC-KP), mostly of Clonal Group 258, representing the majority of strains $[18,19]$.

This study investigated the epidemiology of CRE in a large Italian LTACRF according to different patient categories. The effects of a containment programme, based on screening for CRE carriage and contact isolation plus cohorting of colonized patients, were also evaluated.

\section{Methods}

\section{Setting}

The Don Carlo Gnocchi Foundation is a private non-profit institution offering various rehabilitation programmes (cardiologic, respiratory, orthopaedic, neurologic and postcoma) and operating through several different facilities in Italy (http: / / www. dongnocchi.it). The LTACRF involved in this study had 100 hospital beds organized in different wards of variable intensity of care. The ward characterized by the highest intensity of care [severe brain injuries ward (SBI), 25 beds] receives patients with neurological consequences of acquired brain injuries of traumatic or non-traumatic origin, associated with a coma period (Glasgow Coma Scale $<8$ and duration $>24 \mathrm{~h}$ ) and permanent or temporary impairment of cognitive, physical and psychosocial functions, with an associated altered state of consciousness and a possible or doubtful likelihood of functional and psychosocial recovery. In most cases, these patients are admitted directly from intensive care units or subintensive wards, and require complex rehabilitation practices. This subpopulation is characterized by prolonged length of stay (LOS) (mean \pm standard deviation for 2016: $97 \pm 72$ days), significant comorbidities and high rates of intensive care needs (e.g. mechanical ventilation, ventilator weaning, indwelling device use, advanced wound care, parenteral nutrition and intravenous antibiotic administration).

The other wards (total 75 beds) are medical wards characterized by a medium intensity of care, dealing with the rehabilitation of patients with orthopaedic, cardiologic, respiratory and/or neurologic disorders, who need procedures for functional and/or cognitive rehabilitation. For many of these patients, there is a recent history of acute hospital admission and/or surgery. This subpopulation is characterized by a shorter LOS (mean \pm standard deviation for 2016: $24 \pm 12$ days) and a lower clinical complexity.

\section{Intervention for active surveillance and control of CRE}

Screening for CRE carriage was extended to all patients at admission and, if the patient was found to be negative, was repeated on a weekly basis until the patient was discharged or became colonized. Confirmed CRE carriers were presumed to remain colonized indefinitely and were not subjected to further screening. Patients with a recent history of CRE colonization (positive rectal swab detected within 15 days preceding the LTACRF admission) were considered to be colonized at admission and were not subjected to further screening. Adherence to screening was calculated considering the number of patients who underwent a complete screening schedule vs all admissions. Patients were considered to be non-adherent to screening if: (i) recent history of CRE colonization was unknown, but they were not subjected to screening at admission; or (ii) they had an incomplete screening schedule during the LTACRF stay (e.g. weekly rectal swabs not repeated in a previously negative patient). These patients were excluded from further analysis, and their weeks of admission were not considered for the incidence calculation.

Following admission, while waiting for the screening results, all patients were subjected to pre-emptive contact isolation including contact precautions, admittance to single-bed rooms (whenever possible), and rehabilitation performed inside the bedroom. Confirmed CRE carriers were kept on contact precautions, cohorted in rooms located in specific areas (or in single-bed rooms, whenever possible), and underwent a dedicated rehabilitation programme with restricted access to common gyms (access allowed after all non-colonized patients) or, whenever possible, access to a separate gym for colonized patients.

Procedures for standard infection control precautions and hand hygiene [20] were already in place before the 
intervention commenced, and healthcare workers' adherence to hand hygiene was monitored periodically according to established procedures [21]. All the screening procedures performed and the containment measures adopted were compliant with a nationwide CRE containment strategy recommended by the Italian Ministry of Health since 2013, following the recommendations of the European Centre for Disease Prevention and Control, and were considered among the 'good clinical practices' adopted in Italian healthcare facilities [22,23].

\section{Specimen collection and laboratory methods}

Rectal swabs were collected using the FecalSwab system (Copan, Brescia, Italy) containing $2 \mathrm{~mL}$ of modified Cary-Blair medium, and processed by an external laboratory (Synlab Toscana, Sesto Fiorentino, Italy). The specimens $(10 \mu \mathrm{L}$ of medium) were cultured on chromID CARBA SMART plates (bioMérieux, Marcy L'Etoile, France) to screen for CRE, and on McConkey agar (as a quality control of the rectal swab), within $48 \mathrm{~h}$ of collection. Plates were inspected for growth after $18-24 \mathrm{~h}$ of incubation at $35 \pm 2{ }^{\circ} \mathrm{C}$. Colonies representative of different morphologies grown on the selective medium were identified using the Vitek 2 system (bioMérieux). Phenotypic characterization of carbapenemase production was investigated according to the EUCAST guidelines for detection of resistance mechanisms [24].

In accordance with routine laboratory protocol, blood cultures were performed at the same external laboratory as rectal swab cultures using the BACTEC FX instrument (Becton Dickinson, Franklin Lakes, NJ, USA), and bacterial identification and susceptibility testing were performed using the Vitek 2 system (bioMérieux).

\section{Data collection, outcomes and statistical analysis}

Demographic, admission, discharge and transfer data were obtained from the software used to store and manage patients' data at the Don Carlo Gnocchi Foundation. Microbiological data were obtained from the database of the external laboratory.

The outcomes evaluated included: (i) prevalence of CRE carriage, assessed by a point-prevalence survey (PPS) involving all admitted patients (three PPSs were performed, each over a five-day period; the first was carried out three weeks after the beginning of the intervention, and the others after 6 and 12 months, respectively); and (ii) incidence of in-hospital CRE colonization, defined as positivity of a rectal swab during admission in a patient who was negative at admission screening (expressed as number of patients who acquired CRE colonization per 100 patient-weeks, averaged over the preceding 12 weeks). The prevalence and incidence of CRE colonization were tested for changes (linear and exponential trends) in separate regression models, with the null hypotheses of no change in prevalence or incidence over time during the intervention period. Comparisons between colonization rates in different wards were performed using Chi-squared test with Yates' correction. A P-value $<0.05$ was considered to indicate significance. All analyses were performed using GraphPad software (https://www.graphpad.com). During the study period, CRE bloodstream infections (BSIs) were monitored and notified to local healthcare authorities.

\section{Results}

\section{Intervention for active surveillance and control of CRE at the LTACRF}

The intervention was started on $8^{\text {th }}$ December 2015. At the baseline PPS, carried out from $1^{\text {st }}$ to $5^{\text {th }}$ January 2016, the overall prevalence of CRE carriage among screened patients was $28.4 \%$. All patients were colonized by KPC-KP. The majority of KPC-KP carriers (20/25) were from the SBI ward, where the prevalence of CRE carriage was found to be $90.9 \%$ (20 of 22 patients). In the other wards, the overall prevalence of CRE carriers was 7.5\% (five of 66 screened patients) (Figure 1).

In 2016, a total of 1207 patients were admitted, and the overall adherence to the active surveillance programme was $89.8 \%$. Adherence increased during the study period, with the majority of non-adherent cases concentrated in the first trimester of the study $(47 / 123,38.2 \%)$. Overall, in 2016 , the proportion of patients found to be colonized at admission was $11.6 \%$, and was significantly higher among patients admitted to the $\mathrm{SBI}$ ward than among patients admitted to other wards (44.1\% vs $8.6 \%, P<0.05)$. Over the same period, the proportion of CRE-negative patients that became colonized during the LTACRF stay was $9.9 \%$, revealing a substantial rate of inhospital cross-transmission. Cross-transmission was 10-fold more frequent among patients admitted to the SBI ward than among patients admitted to other wards $(63.5 \%$ vs $6.8 \%$, respectively). The mean length of stay prior to CRE rectal swab positivity was 19 days, and was longer in the SBI ward (23 days) compared with other wards (15 days) (Table I).

In 2016, the prevalence of colonized patients, as evaluated by subsequent PPS, exhibited a decreasing trend (although not significant) in the SBI ward, whilst it remained stable in the other wards (Figure 2). The incidence of in-hospital colonization, averaged over the first trimester of study, was 3.8 per 100 at-risk patient-weeks, and was significantly higher in the SBI ward (17.7) than the other wards $(2.7)(P<0.05)$. In 2016, the incidence of in-hospital colonization exhibited a significant decreasing trend in the SBI ward, and remained stable in the other wards (Figure 3).

In total, seven BSIs caused by CRE were observed during 2016, all among patients that were already colonized. The proportion of colonized patients that developed a CRE BSI was significantly higher in the SBI ward (six of 74 patients, $8.1 \%$ ) than in other wards (one of 147 patients, $0.7 \%)(P<0.05)$. During the study period, all CRE colonizations and BSIs were caused by KPC-KP.

\section{Discussion}

Similar to previous reports from the USA $[12,14,17]$ and Italy [16], this study showed that the dissemination of CRE can heavily involve LTACRFs. These findings, therefore, confirmed the notion that LTACRFs represent an important receptacle for CRE in areas where they have achieved remarkable endemicity. In the study setting, the circulating CRE were KPC-KP, in agreement with the known epidemiology of CRE in Italy $[18,19]$.

Previous studies, however, did not take into consideration the features of different subpopulations of patients admitted to LTACRFs. Regarding this issue, this study showed that the 


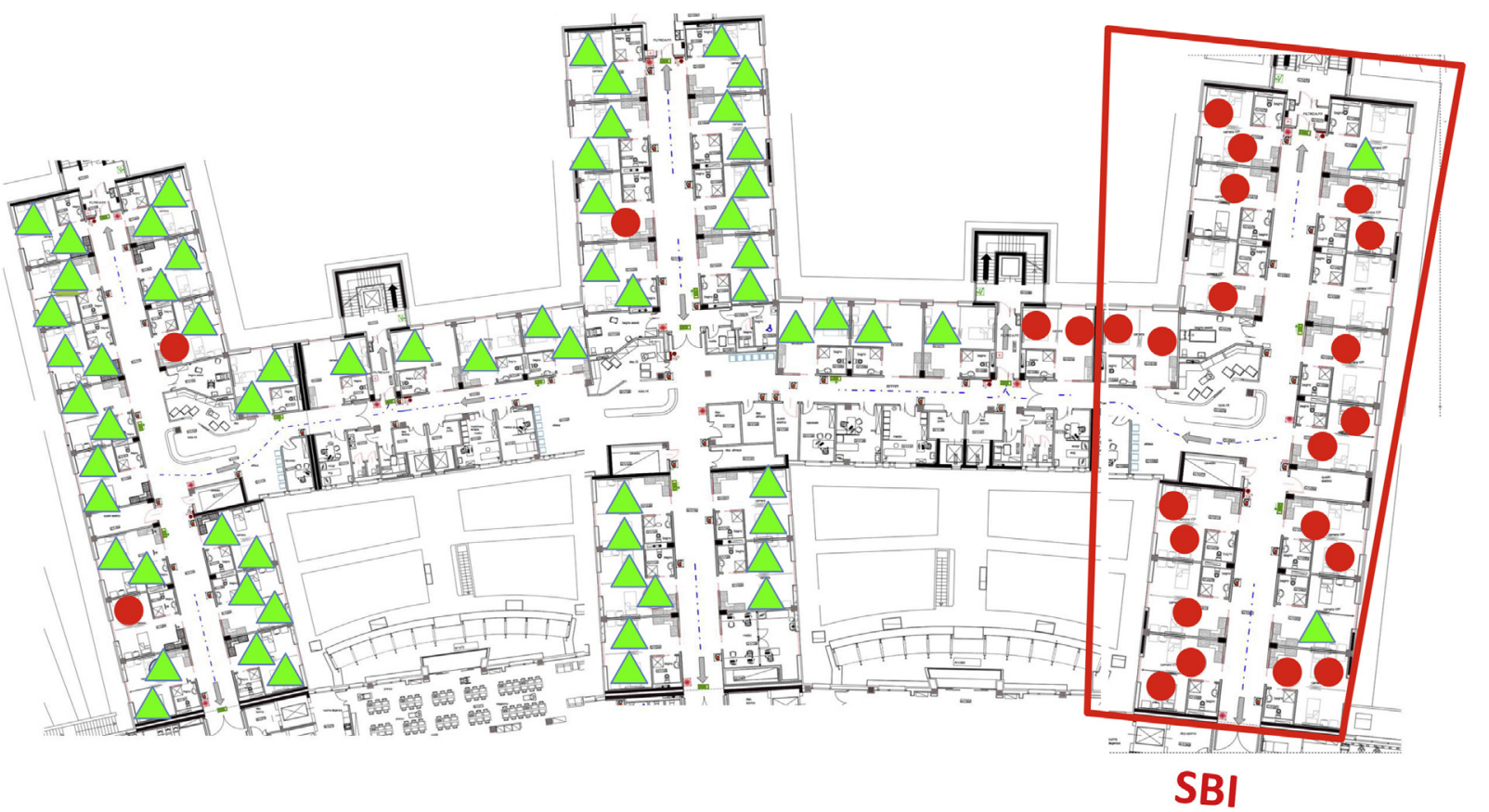

Figure 1. Results of the point prevalence survey for rectal colonization of carbapenemase-producing Enterobacteriaceae performed in January 2016: spatial distribution of colonized (red dot) and non-colonized (green triangle) patients. SBI, severe brain injuries ward.

population of patients admitted to a large LTACRF was not homogeneous in terms of risk for CRE colonization and infection, and found that patients admitted to the SBI ward were at much higher risk, with KPC-KP carriage rates that could reach $90 \%$. This condition was apparently related to both a higher carriage rate at admission and a higher risk for crosscontamination after admission. Considering that patients from SBI wards are characterized by longer LOS and are typically exposed to a higher intensity of care, often including mechanical ventilation, these findings are in overall agreement with previous findings that identified mechanical ventilation and length of stay as two independent risk factors associated with CRE colonization in LTACRFs [12], and a number of features of the population of patients colonized/infected by CRE such as older age, high rates of comorbidities (including renal disease, advanced stage wounds and respiratory failure), and need for indwelling devices (including central venous catheters and tracheostomies) [17].
Regarding CRE containment strategies in LTACRFs, a study carried out in four large LTACRFs in the Chicago metropolitan area has recently shown that a bundled intervention (daily bathing of all patients with cloths impregnated with $2 \%$ chlorhexidine gluconate, surveillance for rectal colonization and contact isolation of positive patients and patients with unknown colonization status, personnel education and adherence monitoring) was able to significantly reduce the prevalence and incidence of CRE colonization over a 12-19-month period. [14] In the present study, the bundle was simpler, only including implementation of a surveillance programme for rectal colonization and standard contact isolation precautions, but results revealed that a similar strategy could be a useful tool for reducing CRE circulation in LTACRF settings. Indeed, a decrease in the prevalence and incidence of CRE colonization was observed in the ward most involved in implementing the intervention. Therefore, these findings support the notion that active surveillance could be important in the control of CRE

Table I

Overview of surveillance data relative to 2016 (data for the first three weeks of surveillance in December 2015 were excluded)

\begin{tabular}{|c|c|c|c|}
\hline & SBI (\%) & Others (\%) & All wards (\%) \\
\hline No. of rectal swabs & 170 & 2269 & 2439 \\
\hline Adherence to screening (\%) & $93 / 106(87.7)$ & $991 / 1101(90)$ & $1084 / 1207(89.8)$ \\
\hline Prevalence of CRE colonization at admission (\%) & $41 / 93(44.1)$ & $85 / 991(8.6)$ & $126 / 1084(11.6)$ \\
\hline Mean interval between admission and colonization (days) & 23 & 15 & 19 \\
\hline No. of reported KPC-KP BSI episodes & 6 & 1 & 7 \\
\hline
\end{tabular}

KPC-KP, Klebsiella pneumoniae carbapenemase-producing K. pneumoniae; CRE, carbapenemase-producing Enterobacteriaceae; BSI, bloodstream infection. 


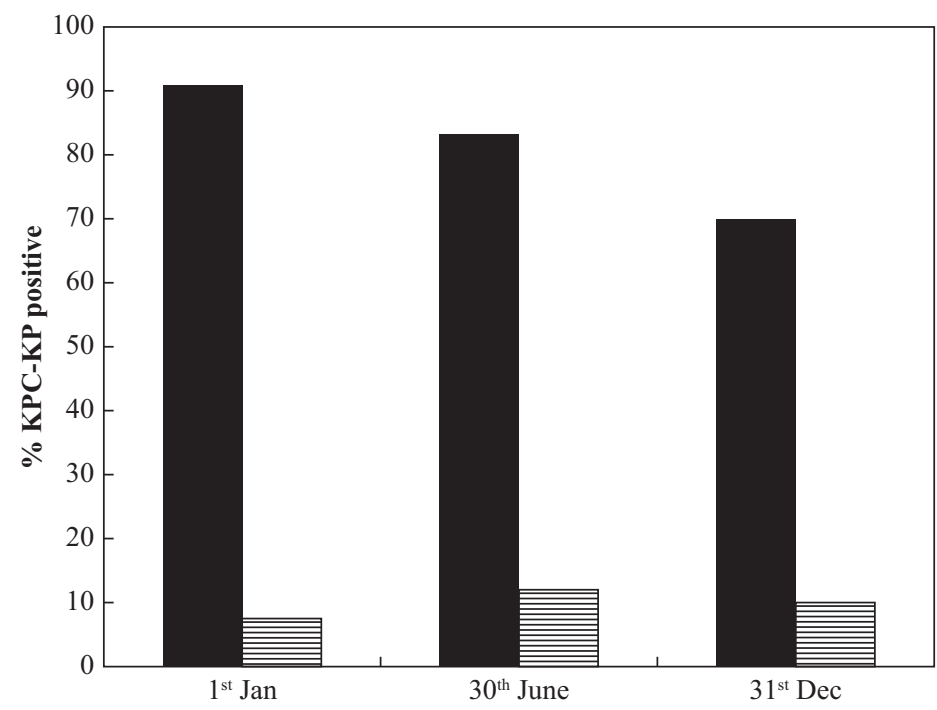

\begin{tabular}{|c|c|c|c|}
\hline Prevalence data on PPSurveys 2016 & $1^{\text {st } J a n}$ & $30^{\text {th }}$ June & $31^{\text {st }}$ Dec \\
\hline Total (\%) & $25 / 88(28.4)$ & $29 / 99(29.3)$ & $25 / 93(26.9)$ \\
\hline SBI (\%) & $20 / 22(90.9)$ & $20 / 24(83.3)$ & $16 / 23(70)$ \\
\hline other wards (\%) & $5 / 66(7.5)$ & $9 / 75(12)$ & $7 / 70(10)$ \\
\hline
\end{tabular}

Figure 2. Semi-annual point prevalence surveys of Klebsiella pneumoniae carbapenemase-producing K. pneumoniae rectal colonization during the study period. Solid bars, severe brain injuries ward (\%); striped bars, other wards (\%).

dissemination in LTACRF settings, as has been shown in other settings $[10,11,22,25]$. However, the impact of the intervention in other wards was limited, and the rate of in-hospital CRE colonization remained high overall, even at the end of the observation period, suggesting that other measures are necessary to further contain the CRE burden in LTACRFs. A deeper analysis of causes leading to breaches in adherence to CRE screening, which averaged $90 \%$, and an evaluation of compliance with standard infection control precautions will be needed.

However, this study has some limitations: (i) although a baseline colonization prevalence assessment was performed,

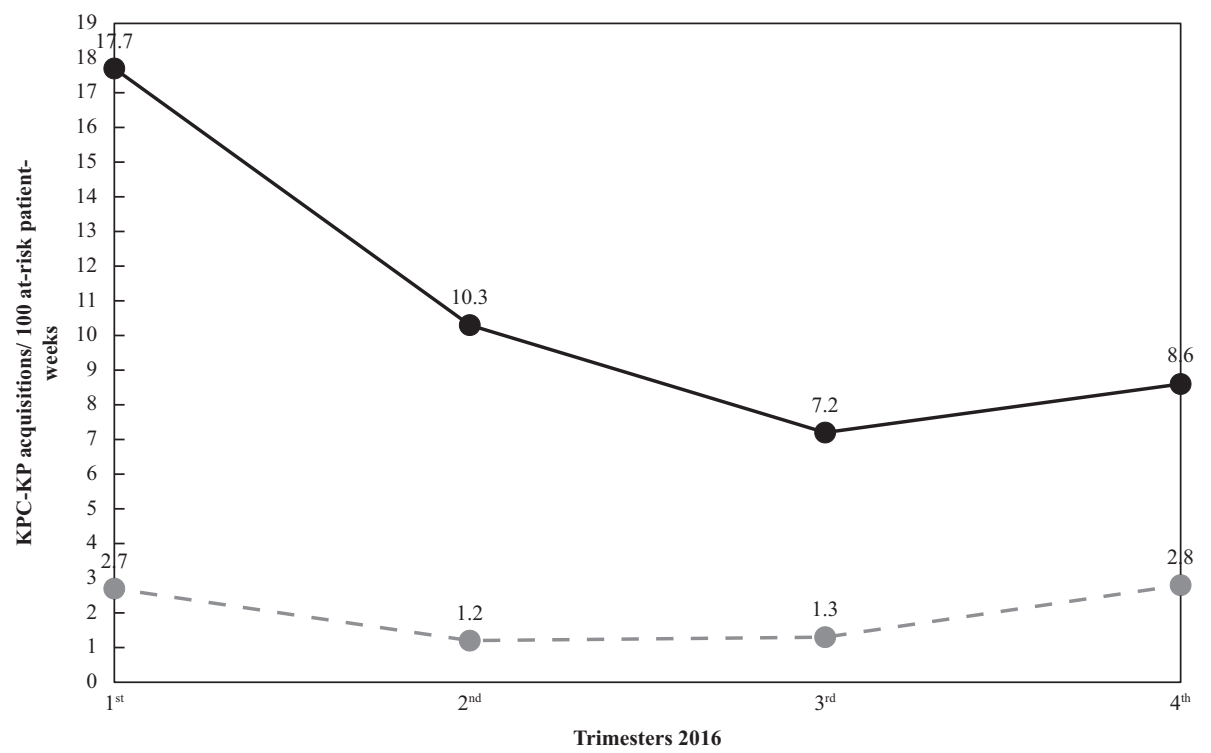

Figure 3. Incidence of Klebsiella pneumoniae carbapenemase-producing K. pneumoniae (KPC-KP) rectal colonization during the study period. Each data point represents the number of patients who acquired KPC-KP per 100 at-risk patient-weeks, averaged over the preceding 12 weeks. Solid line, severe brain injuries ward; dashed line, other wards. 
extensive pre-intervention data are lacking; (ii) compared with previous intervention studies, the study period was relatively short (one year) and a long-lasting observation period could be necessary to confirm the results observed in the first year; and (iii) differences in the genetic and biological features of the KPC-KP circulating strains could have influenced the epidemiologic data observed, and will be the subject of further investigations.

In conclusion, these findings suggest that dissemination of CRE could follow different patterns within the LTACRF setting, depending on the category of patients, which may have relevant implications for infection control policies.

\section{Acknowledgments}

The authors wish to thank Andrea Marella, Alberto Antonelli and Eleonora Riccobono for assistance in data retrieval and storage of bacterial isolates.

\section{Conflict of interest statement None declared.}

\section{Funding sources}

None.

\section{References}

[1] Logan LK, Weinstein RA. The epidemiology of carbapenemresistant Enterobacteriaceae: the impact and evolution of a global menace. J Infect Dis 2017;215:S28-36.

[2] Falagas ME, Tansarli GS, Karageorgopoulos DE, Vardakas KZ. Deaths attributable to carbapenem-resistant Enterobacteriaceae infections. Emerg Infect Dis 2014;20:1170-5.

[3] Rossolini GM. Extensively drug-resistant carbapenemase-producing Enterobacteriaceae: an emerging challenge for clinicians and healthcare systems. J Intern Med 2015;277:528-31.

[4] Gutiérrez-Gutiérrez B, Salamanca E, de Cueto M, Hsueh PR, Viale $\mathrm{P}$, Paño-Pardo JR, et al. A predictive model of mortality in patients with bloodstream infections due to carbapenemaseproducing Enterobacteriaceae. Mayo Clin Proc 2016;91:1362-71.

[5] Papadimitriou-Olivgeris M, Marangos M, Fligou F, Christofidou M, Sklavou C, Vamvakopoulou S, et al. KPC-producing Klebsiella pneumoniae enteric colonization acquired during intensive care unit stay: the significance of risk factors for its development and its impact on mortality. Diagn Microbiol Infect Dis 2013;77:169-73.

[6] Giannella M, Trecarichi EM, De Rosa FG, Del Bono V, Bassetti M, Lewis RE, et al. Risk factors for carbapenem-resistant Klebsiella pneumoniae bloodstream infection among rectal carriers: a prospective observational multicentre study. Clin Microbiol Infect 2014;20:1357-62.

[7] Haverkate MR, Weiner S, Lolans K, Moore NM, Weinstein RA, Bonten MJ, et al. Duration of colonization with Klebsiella pneumoniae carbapenemase-producing bacteria at long-term acute care hospitals in Chicago, Illinois. Open Forum Infect Dis 2016;3:178.

[8] Feldman N, Adler A, Molshatzki N, Navon-Venezia S, Khabra E, Cohen D, et al. Gastrointestinal colonization by KPC-producing Klebsiella pneumoniae following hospital discharge: duration of carriage and risk factors for persistent carriage. Clin Microbiol Infect 2013;19:E190-6.

[9] Zimmerman FS, Assous MV, Bdolah-Abram T, Lachish T, Yinnon AM, Wiener-Well Y. Duration of carriage of carbapenemresistant Enterobacteriaceae following hospital discharge. Am J Infect Contr 2013;41:190-4.
[10] Tacconelli E, Cataldo MA, Dancer SJ, De Angelis G, Falcone M, Frank $\mathrm{U}$, et al. ESCMID guidelines for the management of the infection control measures to reduce transmission of multidrugresistant Gram-negative bacteria in hospitalized patients. Clin Microbiol Infect 2014;20(Suppl. 1):1-55.

[11] French CE, Coope C, Conway L, Higgins JPT, McCulloch J, Okoli G, et al. Control of carbapenemase-producing Enterobacteriaceae outbreaks in acute settings: an evidence review. J Hosp Infect 2017;95:3-45.

[12] Lin MY, Lyles-Banks RD, Lolans K, Hines DW, Spear JB, Petrak R, et al. The importance of long-term acute care hospitals in the regional epidemiology of Klebsiella pneumoniae carbapenemaseproducing Enterobacteriaceae. Clin Infect Dis 2013;57:1246-52.

[13] Marchaim D, Chopra T, Pogue JM, Perez F, Hujer AM, Rudin S, et al. Outbreak of colistin-resistant, carbapenem-resistant Klebsiella pneumoniae in metropolitan Detroit, Michigan. Antimicrob Agents Chemother 2011;55:593-9.

[14] Hayden MK, Lin MY, Lolans K, Weiner S, Blom D, Moore NM, et al. Prevention of colonization and infection by Klebsiella pneumoniae carbapenemase-producing Enterobacteriaceae in long-term acute-care hospitals. Clin Infect Dis 2015;60:1153-61.

[15] Marchaim D, Chopra T, Bogan C, Bheemreddy S, Sengstock D, Jagarlamudi R, et al. The burden of multidrug-resistant organisms on tertiary hospitals posed by patients with recent stays in longterm acute care facilities. Am J Infect Contr 2012;40:760-5.

[16] Rossini A, Di Santo SG, Libori MF, Tiracchia V, Balice MP, Salvia A. Risk factors for carbapenemase-producing Enterobacteriaceae colonization of asymptomatic carriers on admission to an Italian rehabilitation hospital. J Hosp Infect 2016;92:78-81.

[17] Han JH, Goldstein EJC, Wise J, Bilker WB, Tolomeo P, Lautenbach E. Epidemiology of carbapenem-resistant Klebsiella pneumoniae in a network of long-term acute care hospitals. Clin Infect Dis 2017;64:839-44.

[18] Giani T, Pini B, Arena F, Conte V, Bracco S, Migliavacca R, et al. Epidemic diffusion of KPC carbapenemase-producing Klebsiella pneumoniae in Italy: results of the first countrywide survey, 15 May to 30 June 2011. Euro Surveill 2013;18. pii:20489.

[19] Conte V, Monaco M, Giani T, D’Ancona F, Moro ML, Arena F, et al. Molecular epidemiology of KPC-producing Klebsiella pneumoniae from invasive infections in Italy: increasing diversity with predominance of the ST512 clade II sublineage. J Antimicrob Chemother 2016;71:3386-91.

[20] Zingg W, Holmes A, Dettenkofer M, Goetting T, Secci F, Clack L, et al. Hospital organisation, management, and structure for prevention of health-care-associated infection: a systematic review and expert consensus. Lancet Infect Dis 2015;15:212-24.

[21] World Health Organization. WHO guidelines on hand hygiene in health care. First global patient safety challenge: clean care is safer care. Geneva: World Health Organization; 2009.

[22] European Centre for Disease Prevention and Control. Risk assessment on the spread of carbapenemase-producing Enterobacteriaceae (CPE) through patient transfer between healthcare facilities, with special emphasis on cross-border transfer. Stockholm: ECDC; 2011.

[23] Ministero della Salute. Circolare 'Sorveglianza e controllo delle infezioni da batteri produttori di carbapenemasi (CPE)'. Circular letter 'Surveillance and control of infections due to carbapenemase-producing bacteria (CPE)'. Roma: Ministero della Salute; 2013.

[24] Giske CG. EUCAST guidelines for detection of resistance mechanisms and specific resistances of clinical and/or epidemiological importance. 2013. p. 1-40. http://www.eucast.org/fileadmin/ src/media/PDFs/EUCAST_files/Resistance_mechanisms/EUCAST_ detection_of_resistance_mechanisms_v1.0_20131211.pdf.

[25] Campos AC, Albiero J, Ecker AB, Kuroda CM, Meirelles LE, Polato A, et al. Outbreak of Klebsiella pneumoniae carbapenemaseproducing K. pneumoniae: a systematic review. Am J Infect Control 2016;44:137480. 